\title{
Neumonía grave causada por Haemophilus influenzae no tipificable en un lactante: Reporte de un caso
}

\author{
LUIS E. VEGA-BRICEÑO, CECILIA PERRET P., NILS HOLMGREN R. e IGNACIO SÁNCHEZ D.
}

\section{Non-typable Haemophilus influenzae severe pneumonia in an infant: Case report}

Haemophilus influenzae $b$ (Hib) disease used to be a frequent cause of pneumonia in children. After the widespread use of Hib conjugate vaccines, non-b H. influenzae have become an important cause of pneumonia. We present the case of an infant with severe pneumonia with empyema and bacteremia after he had completed the Hib vaccination schedule. Non typable $H$. influenzae was recovered from blood and pleural effusion cultures. We review the literature about this unusual clinical presentation in our community and alert about the possible emergence of non typable $H$. influenzae as a frequent cause of community-acquired pneumonia in children.

Key words: Severe pneumonia, empyema, children, non typable Haemophilus influenzae

Palabras claves: Neumonía grave, empiema, niños, $H$. influenzae no tipificable

\section{Introducción}

La neumonía bacteriana es aún una importante causa de morbimortalidad infantil en el mundo. En países subdesarrollados, más de 4 millones de niños menores de 5 años mueren anualmente por esta causa $^{1}$; más alarmante es el hecho que muchas de estas muertes en niños son prevenibles con la vacunación.

Haemophilus influenzae tipo b (Hib) tiene un amplio espectro de expresiones clínicas en la edad pediátrica: epiglotitis, traqueitis, bronquitis, neumonía, meningitis, artritis, celulitis y pericarditis. En países con baja cobertura de vacunación, más de $90 \%$ de las infecciones invasoras asignables a $H$. influenzae pertenecen al serotipo $\mathrm{b}^{2}$, mientras que en aquellos con una alta tasa de vacunación esto variará. La introducción de la vacuna conjugada contra $\mathrm{Hib}$ en nuestro medio en 1996, demostró una efectividad superior al $80 \%$ en la reducción de las neumonía bacteriémicas causadas por $\mathrm{Hib}^{3,4}$.
Cepas no tipificables (nt) de $H$. influenzae se han revelado como una importante causa de infección respiratoria baja en niños ${ }^{5}$. En países de África numerosos casos de neumonía en niños son causadas por estas cepas $\mathrm{nt}^{6,7}$; aunque existen variaciones entre los estudios, cerca del $60 \%$ de las muestras obtenidas por punción pulmonar arrojaron el aislamiento de cepas de $H$. influenzae $\mathrm{nt}^{8-10}$. Hay que enfatizar que estas formas de infección por $H$. influenzae nt no son prevenibles por la vacuna anti $H i b$.

Nuestro objetivo fue presentar un caso infrecuente de pleuroneumonía causado por una cepa de $H$. influenzae nt, alertando sobre la eventual emergencia de estas cepas como causantes de neumonía adquirida en la comunidad en pacientes pediátricos en nuestro medio.

\section{Caso clínico}

Lactante de sexo femenino, con 16 meses de edad, sin antecedentes mórbidos, consultó por

Departamento de Pediatría. Pontificia Universidad Católica de Chile, Santiago, Chile.

Becado del Programa de Enfermedades Respiratorias del Niño. (LV-B)

Centro de Investigaciones Médicas. (CP, NH)

Sección Respiratorio. (IS)

Recibido: 21 octubre 2004

Aceptado: 21 diciembre 2004 
presentar coriza, tos y fiebre dos día antes, indicándosele antipiréticos y terapia broncodilatadora. Cinco días más tarde se agregó compromiso del estado general con vómitos y dificultad respiratoria por lo que acudió al Servicio de Emergencia, donde se constató la fiebre $\left(39,5^{\circ} \mathrm{C}\right.$ axilar), aumento del trabajo respiratorio y una saturación de oxígeno de $91 \%$ (con $\mathrm{FiO}_{2}$ ambiental), decidiéndose su hospitalización. Destacaba al ingreso una FC: 196/min, FR: 56/min, decaimiento y dificultad respiratoria moderada, además de abolición de los sonidos pulmonares en el hemitórax izquierdo con broncofonía evidente. El resto del examen físico era normal.

Los exámenes de laboratorio revelaron un hematocrito de 29\%, hemoglobina: $9,6 \mathrm{~g} / \mathrm{dl}$, leucocitos: $18.800 / \mathrm{mm}^{3}$ (baciliformes $25 \%$ ), VHS: $99 \mathrm{~mm} / \mathrm{h}$, PCR: 18,2mg/dl ( $<1,0 \mathrm{mg} / \mathrm{dl})$. La radiografía de tórax al momento de ingresar comprobó la presencia de una consolidación pulmonar basal izquierda y efusión pleural adyacente (Figura 1). El líquido pleural mostró leucocitos 9000/ $\mathrm{mm}^{3}$, con predominio polimor-fonuclear $(97 \%)$, glucosa: $14 \mathrm{mg} / \mathrm{dl}$, proteínas: 5,4 mg/dl, $\mathrm{pH}$ : 7,03 y LDH: 670 U/L. La tinción de Gram directa comprobó la presencia de cocobacilos gramnegativos por lo que se procedió al drenaje pleural extrayéndose inicialmente $170 \mathrm{ml}$ de exudado. Se inició tratamiento endovenoso con cefotaxima y cloxacilina; al cabo de 48 horas se informó la presencia de $H$. influenzae no productor de $\beta$ lactamasa tanto en el líquido pleural como en

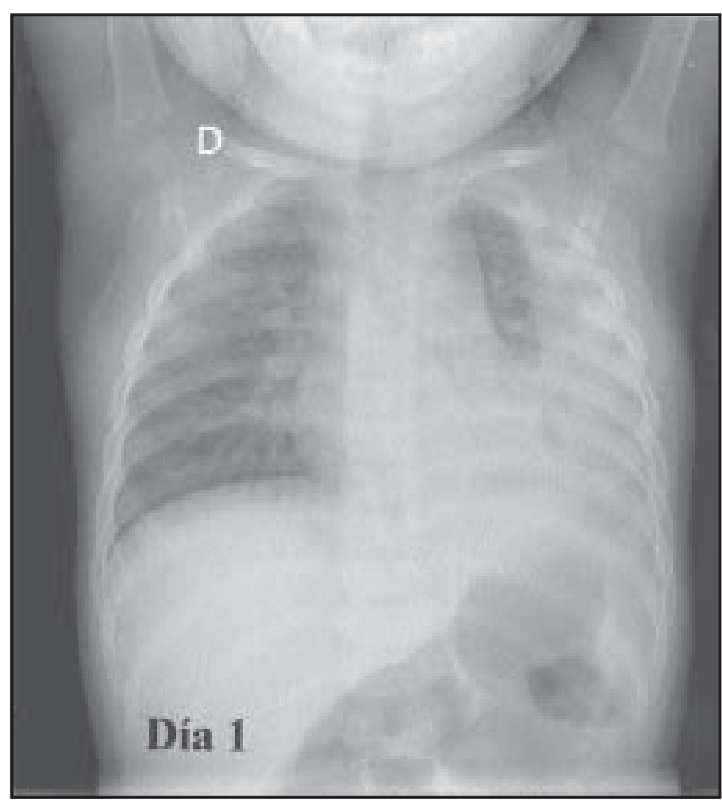

Figura 1. Radiografía de tórax al ingreso. Consolidación del lóbulo inferior izquierdo con presencia de efusión pleural ipsilateral. sangre, decidiéndose suspender la terapia antiestafilocóccica. La evolución clínica posterior fue favorable, haciéndose afebril a las 48 horas, momento en que un control de laboratorio mostró hematocrito: $25 \%$, hemoglobina: $8,2 \mathrm{gr} / \mathrm{dl}$, leucocitos: $9.500 / \mathrm{mm}^{3}$, sin desviación a izquierda, VHS: $69 \mathrm{~mm} / \mathrm{h}$, PCR: 11,8mg/dl. La niña recibió oxígeno durante las primeras 24 horas ( $\mathrm{FiO}_{2}$ máximo 35\%). La cepa de $H$. influenzae fue enviada al laboratorio de referencia del Instituto de Salud Pública de Chile donde se confirmó que se trataba de una cepa no tipificable mediante un screening de coaglutinación (Phadebact ${ }^{\circledR}$ ) que empleó un anticuerpo policlonal, y luego antisueros específicos (DIFCO) para Hib y cada uno de los otros cinco serotipos (a, c, d, e, f). La radiografía de tórax al quinto día de hospitalización, pronto al alta, comprobó la resolución subtotal del proceso neumónico (Figura 2) decidiéndose continuar terapia ambulatoria con amoxicilina hasta completar 14 días. En un control a los 30 días la niña estaba asintomática, en un excelente estado general, y un control radiográfíco de tórax fue normal.

\section{Discusión}

Reportamos el caso de una lactante previamente vacunada contra Hib que desarrolló una forma grave e inusual de pleuroneumonía causada por una cepa de $H$. influenzae nt. Resaltamos

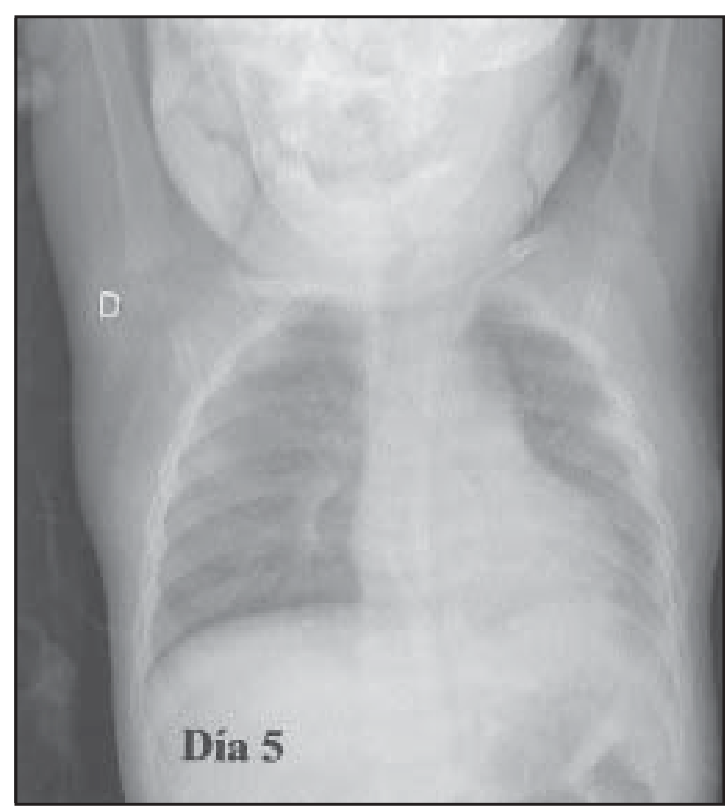

Figura 2. Radiografía de tórax al egreso. Resolución importante de la consolidación del lóbulo inferior izquierdo con una re-expansión pulmonar significativa. 
que las cepas de $H$. influenzae nt pueden ser invasoras y producir infecciones graves en niños, en ausencia de factores de riesgo identificables. Bajanca y cols revelaron un incremento significativo de los serotipos no b y cepas nt en Portugal, desde 19 hasta $80 \%$, en forma simultánea a la disminución del serotipo $\mathrm{b}^{11}$. Debe considerarse que en la era prevacuna, la meningitis representó hasta el $70 \%$ de la enfermedad por $\mathrm{Hib}$ observada, seguida muy de cerca por la epiglotitis en los países anglosajones, mientras que las cepas $H$. influenzae no b se asocian más frecuentemente con la presencia de bacteriemia y neumonía en niños ${ }^{2}$.

La neumonía sigue siendo la forma más frecuente de presentación de $H$. influenzae en los países subdesarrollados, siendo difícil determinar la etiología en muchas neumopatías. Se estima que en Sudamérica, 5 a $50 \%$ de las neumonías son producidas por $H$. influenzae ${ }^{12}$. Nohynek $\mathrm{y}$ cols mostraron que $H$. influenzae no b y Streptococcus pneumoniae fueron los agentes bacterianos más frecuen-temente identificados en una serie de pacientes hospitalizados por neumonías adquiridas en la comunidad ${ }^{13}$. Por otra parte, en el grupo de niños vacunados contra $\mathrm{Hib}$ se describe un incremento relativo de la enfermedad causada por $H$. influenzae no b. Entre $10 \mathrm{y}$ $100 \%$ de los niños sanos pueden portar $H$. influenzae en la faringe; $75-95 \%$ de ellos corresponden a cepas no tipificables y menos de un cuarto portan las cepas capsuladas ${ }^{14}$. Diversas observaciones sugieren que la mayoría de casos de neumonía en países subdesarrollados está relacionada con la aspiración de secreciones nasofaríngeas. Un estudio en Bangladesh reveló que $76 \%$ de las neumonías producidas por $H$. influenzae eran causadas por cepas $\mathrm{nt}^{10,15}$. Es probable que los cultivos de sangre subestimen el verdadero rol de las cepas nt que son menos bacteriémicas. Tres estudios etiológicos de neumonía efectuados en el tercer mundo mediante punciones pulmonares revelaron que las cepas nt de $H$. influenzae se aislaron en más del $50 \%$ de las veces ${ }^{6,716}$. Mas aún, algunas veces las cepas nt aglutinan espontáneamente, pudiendo ser incorrectamente identificadas como cepas del serotipo $b^{10}$.

Es necesario desarrollar vacunas contra $H$. influenzae nt con el objeto de ampliar la protección contra las neumonías bacterianas. La utilidad de la vacunación puede estar subestimado si se minimiza la existencia de formas graves de neumonía causadas por estas cepas. Finalmente, existiendo formas graves de neumonía en la comunidad producidas por las cepas nt, debe con- siderarse esta posible etiología al momento de iniciar una antibioterapia empírica. Alertamos a la comunidad médica sobre la posibilidad de la emergencia de infecciones causadas por $H$. influenzae no $b$ y en particular cepas nt. Futuras series prospectivas podrán evaluar la magnitud o importancia de estos agentes como responsables de neumonía en la comunidad.

\section{Resumen}

Previo a la introducción de la vacuna conjugada, Haemophilus influenzae b (Hib) representó una de las causas importantes de neumonía en niños. Recientemente cepas no b y no tipificable (nt) han emergido como agentes importantes de enfermedad. Reportamos el caso de una lactante afectada por una neumonía consolidante extensa con empiema y bacteriémica, con el antecedente de haber recibido antes vacuna contra $\mathrm{Hib}$. Los cultivos de sangre y líquido pleural revelaron la presencia de $H$. influenzae nt. La evolución clínica fue favorable, retirándose el drenaje pleural al segundo día. Fue tratada con cefotaxima endovenosa durante cinco días y luego 9 días de amoxicilina en forma ambulatoria. Se revisa la literatura acerca de esta inusual forma de presentación en nuestro medio y se advierte la emergencia de cepas no tipificables de $H$. influenzae como responsables de algunas formas graves de neumonía.

\section{Bibliografía}

1.- World Health Organization. WHO Recommended Surveillance Standards. Acute Lower Respiratory Infections (aLTI) and Pneumonia. WHO/CDC/CSRI/ SR/99.2. Geneva: WHO, 1999.

2.- Heath P T, Booy R, Azzopardi H J, Slack M P, Fogarty J, Moloney A C, et al. Non-type b Haemophilus influenzae disease: clinical and epidemiologic characteristics in the Haemophilus influenzae type $\mathrm{b}$ vaccine era. Pediatr Infect Dis J 2001; 20: 300-5.

3.- Levine O S, Lagos R, Muñoz A, Villaroel J, Alvarez A M, Abrego P, et al. Defining the burden of pneumonia in children preventable by vaccination against Haemophilus influenzae type b. Pediatr Infect Dis J 1999; 18: 1060-4.

4.- Lagos R, Horwitz I, Toro J, San Martín O, Abrego P, Bustamante $\mathrm{C}$, et al. Large scale, postlicensure, selective vaccination of Chilean infants with PRP-T conjugate vaccine: practicality and effectiveness in preventing invasive Haemophilus influenzae type $\mathrm{b}$ infections. Pediatr Infect Dis J 1996; 15: 216-22.

5.- Wallace R J Jr, Musher D M, Septimus E J, McGowan J E Jr, Quinones FJ, Wiss K, et al. Haemophilus influenzae infections in adults: characterization of strains by serotypes, biotypes, and beta-lactamase production. J Infect Dis 1981; 144: 101-6.

6.- Adegbola R A, Falade A G, Sam B E, Aidoo M, Baldeh I, Hazlett D, et al. The etiology of pneumonia in malnourished and well-nourished Gambian children. 
Pediatr Infect Dis J 1994; 13: 975-82.

7.- Shann F, Gratten M, Germer S, Linnemann V, Hazlett D, Payne R. Aetiology of pneumonia in children in Goroka Hospital, Papua New Guinea. Lancet 1984; 2: 537-41.

8.- Weinberg G A, Ghafoor A, Ishaq Z, Nomani N K, Kabeer M, Anwar F, et al. Clonal analysis of Hemophilus influenzae isolated from children from Pakistan with lower respiratory tract infections. J Infect Dis 1989; 160: 634-43.

9.- Straus W L, Qazi S A, Kundi Z, Nomani N K, Schwartz B. Antimicrobial resistance and clinical effectiveness of co-trimoxazole versus amoxycillin for pneumonia among children in Pakistan: randomised controlled trial. Pakistan Co-trimoxazole Study Group. Lancet 1998; 352: 270-4.

10.- Shann F. Haemophilus influenzae pneumonia: type b or non-type b? Lancet 1999; 354: 1488-90.

11.- Bajanca P, Canica M. Multicenter Study Group. Emergence of nonencapsulated and encapsulated non-b-type invasive Haemophilus influenzae isolates in Portugal
(1989-2001). J Clin Microbiol 2004; 42: 807-10.

12.- de Andrade A L, de Andrade J G, Martelli C M, e Silva S A, de Oliveira R M, Costa M S, et al. Effectiveness of Haemophilus influenzae b conjugate vaccine on childhood pneumonia: a case-control study in Brazil. Int J Epidemiol 2004; 33: 173-81.

13.- Nohynek H, Eskola J, Laine E, Halonen P, Ruutu P, Saikku P, et al. The causes of hospital-treated acute lower respiratory tract infection in children. Am J Dis Child 1991; 145: 618-22.

14.- Moxon E R, Wilson R. The role of Haemophilus influenzae in the pathogenesis of pneumonia. Rev Infect Dis 1991; 13 Suppl 6: S518-27.

15.- Utsunomiya Y, Ahmed K, Rikitomi N, Ruhulamin M, Hanif M, Masaki $\mathrm{H}$, et al. Isolation of pathogenic bacteria from induced sputum from hospitalized children with pneumonia in Bangladesh. J Trop Pediatr 1998; 44: 338-42.

16.- Wall R A, Corrah P T, Mabey D C, Greenwood B M. The etiology of lobar pneumonia in the Gambia. Bull World Health Organ 1986; 64: 553-8.

Correspondencia a:

Luis E. Vega-Briceño

levega@puc.cl 